\title{
ON HYPERBOLICITY AND TAUTNESS MODULO AN ANALYTIC SUBSET OF HARTOGS DOMAINS
}

\author{
DO DUC THAI, PASCAL J. THOMAS, NGUYEN VAN TRAO, AND MAI ANH DUC
}

(Communicated by Franc Forstneric)

\begin{abstract}
Let $X$ be a complex space and $H$ a positive homogeneous plurisubharmonic function $H$ on $X \times \mathbb{C}^{m}$. Consider the Hartogs-type domain $\Omega_{H}(X):=$ $\left\{(z, w) \in X \times \mathbb{C}^{m}: H(z, w)<1\right\}$. Let $S$ be an analytic subset of $X$. We give necessary and sufficient conditions for hyperbolicity and tautness modulo $S \times \mathbb{C}^{m}$ of $\Omega_{H}(X)$, with the obvious corollaries for the special case of Hartogs domains.
\end{abstract}

\section{INTRODUCTION}

Let $X$ be a complex space and $\varphi: X \rightarrow[-\infty, \infty)$ be an upper semicontinuous function on $X$. The Hartogs domain

$$
\Omega_{\varphi}(X):=\left\{(x, z) \in X \times \mathbb{C}:|z|<e^{-\varphi(x)}\right\}
$$

is a classical object in several complex variables. In particular, during the past ten years much attention has been given to the properties of Hartogs domains from the viewpoint of hyperbolic complex analysis. For instance, in [15], the authors obtained necessary and sufficient conditions for the hyperbolicity and the tautness of $\Omega_{\varphi}(X)$. We refer readers to the articles [14, 4], and the references therein for the development of related subjects.

More generally, if $H: X \times \mathbb{C}^{m} \rightarrow[-\infty, \infty)$ is an upper semicontinuous function such that $H(z, w) \geq 0, H(z, \lambda w)=|\lambda| H(z, w), \lambda \in \mathbb{C}, z \in X, w \in \mathbb{C}^{m}$, we put

$$
\Omega_{H}(X):=\left\{(z, w) \in X \times \mathbb{C}^{m}: H(z, w)<1\right\}
$$

and call it a Hartogs type domain [16. Hartogs domains correspond to the special case $m=1$ and $H(z, w)=|w| e^{\varphi(z)}$.

Motivated by studying hyperbolicity and tautness modulo an analytic subset of complex spaces, the main goal of this article is to give necessary and sufficient conditions on hyperbolicity or tautness modulo a "vertical" analytic subset of the Hartogs domains $\Omega_{\varphi}(X)$. The results are given in Section 2 but first we recall some basic notions.

Received by the editors December 18, 2011 and, in revised form, January 6, 2012.

2010 Mathematics Subject Classification. Primary 32F45; Secondary 32C25, 32H25, 32Q45.

Key words and phrases. Hyperbolicity modulo an analytic subset, tautness modulo an analytic subset, Hartogs domains.

The research of the authors was supported by an NAFOSTED grant of Vietnam and by an ARCUS cooperation program with the support of the Régions Ile-de-France and Midi-Pyrénées. 
Definition 1.1 (See [11, p. 68]). Let $X$ be a complex space and $S$ be an analytic subset of $X$. We say that $X$ is hyperbolic modulo $S$ if for every pair of distinct points $p, q$ of $X$ we have $d_{X}(p, q)>0$ unless both are contained in $S$, where $d_{X}$ is the Kobayashi pseudodistance of $X$.

If $S=\emptyset$, then $X$ is said to be hyperbolic.

Definition 1.2 (See [11, p. 240]). Let $X$ be a complex space and $S$ be an analytic subset in $X$. We say that $X$ is taut modulo $S$ if it is normal modulo $S$; i.e., for every sequence $\left\{f_{n}\right\}$ in $\operatorname{Hol}(\mathbb{D}, X)$ one of the following holds:

(i) There exists a subsequence of $\left\{f_{n}\right\}$ which converges uniformly to $f \in$ $\operatorname{Hol}(\mathbb{D}, X)$ in $\operatorname{Hol}(\mathbb{D}, X)$.

(ii) The sequence $\left\{f_{n}\right\}$ is compactly divergent modulo $S$ in $\operatorname{Hol}(\mathbb{D}, X)$; i.e., for each compact set $K \subset \mathbb{D}$ and each compact set $L \subset X \backslash S$, there exists an integer $N$ such that $f_{n}(K) \cap L=\emptyset$ for all $n \geq N$.

If $S=\emptyset$, then $X$ is said to be taut. It is immediate from the definition that if $S \subset S^{\prime} \subset X$ and $X$ is taut modulo $S$, then it is taut modulo $S^{\prime}$, so in particular if $X$ is taut, it is taut modulo $S$ for any analytic subset $S$.

Of course, the converse does not hold.

Example 1.3. Let $X=\left\{(z, w) \in \mathbb{C}^{2}|| z|<1| z w \mid,<1\right\}$ and $S:=\{0\} \times \mathbb{C}$. Then:

(i) $X$ is not hyperbolic, but $X$ is hyperbolic modulo $S$.

(ii) $X$ is not taut, but $X$ is taut modulo $S$.

(iii) $X \backslash S$ is taut (thus hyperbolic).

We could have $X \backslash S$ be taut without $X$ being taut modulo $S$; for instance, $\mathbb{C} \backslash\{0,1\}$ is taut, but $\mathbb{C}$ is not taut modulo $\{0,1\}$.

On the other hand, there are examples of domains taut modulo $S$ such that $X \backslash S$ is not taut: just take $X$ a taut domain and $S$ such that the codimension of $S$ is at least 2. Then $X \backslash S$ is not pseudoconvex, therefore not taut.

Proof of Example 1.3. Since the complex line $S$ is contained in $X$, it cannot be hyperbolic, thus not taut either. On the other hand, $X \backslash S$ is biholomorphic to $(\mathbb{D} \backslash\{0\}) \times \mathbb{D}$ under the map $(z, w) \mapsto(z, z w)$, and the latter is clearly taut, which proves (iii).

Now suppose $\left(z_{0}, w_{0}\right) \neq\left(z_{1}, w_{1}\right) \in X$, with at least one of them not in $S$. If $z_{0} \neq z_{1}$, then $d_{X}\left(\left(z_{0}, w_{0}\right),\left(z_{1}, w_{1}\right)\right) \geq d_{\mathbb{D}}\left(z_{0}, z_{1}\right)>0$; if $z_{0}=z_{1}$, then $z_{0} \neq 0$. Given any finite set of points of $X,\left(\zeta_{k}, \eta_{k}\right)$ which connect $\left(z_{0}, w_{0}\right)$ to $\left(z_{1}, w_{1}\right)$ via consecutive analytic disks, either there is some $k$ such that $\left|\zeta_{k}\right| \leq\left|z_{0}\right| / 2$ and then the corresponding sum will contribute at least $d_{\mathbb{D}}\left(z_{0}, z_{0} / 2\right)$ or there is not, and then all points are in $X \cap\left\{|z|>\left|z_{0}\right| / 2\right\} \subset\left\{\left|z_{0}\right| / 2<|z|<1,|w|<2 /\left|z_{0}\right|\right\}:=P$ and the sum will be bounded below by $d_{P}\left(\left(z_{0}, w_{0}\right),\left(z_{1}, w_{1}\right)\right) \geq \frac{2}{\left|z_{0}\right|} d_{\mathbb{D}}\left(w_{0}, w_{1}\right)>0$. So $X$ is hyperbolic modulo $S$.

Proof of (ii). Assume that $\left\{f_{n}\right\} \subset \operatorname{Hol}(\mathbb{D}, X)$ is not compactly divergent modulo $S$. Write $f_{n}=\left(g_{n}, h_{n}\right)$ with $g_{n}, h_{n} \in \operatorname{Hol}(\mathbb{D}, \mathbb{C})$ satisfying $\left|g_{n}(z)\right|<1,\left|g_{n}(z) h_{n}(z)\right|<1$ for all $z \in \mathbb{D}$ and for $n=1,2, \cdots$. Since $\left\{f_{n}\right\}$ is not compactly divergent modulo $S,\left\{g_{n}\right\}$ is not compactly divergent modulo $\{0\}$. Therefore, by Montel's theorem 
we may assume, without loss of generality, that $\left\{g_{n}\right\}$ converges uniformly on every compact subset of $\mathbb{D}$ to a holomorphic function $g \in \operatorname{Hol}(\mathbb{D}, \mathbb{D})$, not identically zero. Since $\left\{g_{n} h_{n}\right\}$ is not compactly divergent on $\mathbb{D}$ and since $\mathbb{D}$ is taut, taking a subsequence we may assume that $\left\{g_{n} h_{n}\right\}$ also converges uniformly on every compact subset of $\mathbb{D}$ to a holomorphic function $\gamma \in \operatorname{Hol}(\mathbb{D}, \mathbb{C})$. Hence $\left\{h_{n}\right\}$ converges uniformly on every compact subset of $\mathbb{D}$ to a meromorphic function $h:=\gamma / g$ on $\mathbb{D}$. Moreover, by Hurwitz's theorem $h$ is actually holomorphic on $\mathbb{D}$, and thus $\left\{f_{n}\right\}$ converges uniformly on every compact subset of $\mathbb{D}$ to a holomorphic map $f:=(g, h) \in \operatorname{Hol}(\mathbb{D}, \bar{X})$.

We now prove that $f \in \operatorname{Hol}(\mathbb{D}, X)$. Since $g \in \operatorname{Hol}(\mathbb{D}, \mathbb{D})$, it suffices to show that $|g(z) h(z)|<1$ for all $z \in \mathbb{D}$. Indeed, suppose not. Then there is $z_{0} \in \mathbb{D}$ such that $\left|g\left(z_{0}\right) h\left(z_{0}\right)\right|=1$. By the maximum principle, $g h$ is a constant function. Therefore, $|g(z) h(z)|=1$ for every $z \in \mathbb{D}$. This is not possible because $\left\{g_{n} h_{n}\right\} \subset \operatorname{Hol}(\mathbb{D}, \mathbb{D})$ is not compactly divergent. Thus, the proof is complete.

\section{MAIN RESUltS}

We denote $\widetilde{S}:=S \times \mathbb{C}^{m}$. Recall that $H: X \times \mathbb{C}^{m} \rightarrow[-\infty, \infty)$ is an upper semicontinuous function such that $H(z, w) \geq 0, H(z, \lambda w)=|\lambda| H(z, w), \lambda \in \mathbb{C}, z \in$ $X, w \in \mathbb{C}^{m}$.

Theorem 2.1. Let $X$ be a complex space and $S$ be an analytic subset in $X$. Then $\Omega_{H}(X)$ is hyperbolic modulo $\widetilde{S}$ if and only if $X$ is hyperbolic modulo $S$ and the function $H$ satisfies the following condition:

$$
\begin{aligned}
& \text { If }\left\{z_{k}\right\}_{k \geq 1} \subset X \backslash S \text { with } \lim _{k \rightarrow \infty} z_{k}=z_{0} \in X \backslash S \\
& \quad \text { and }\left\{w_{k}\right\}_{k \geq 1} \subset \mathbb{C}^{m} \text { with } \lim _{k \rightarrow \infty} w_{k}=w_{0} \neq 0 \text {, then } \liminf _{k \rightarrow \infty} H\left(z_{k}, w_{k}\right) \neq 0 .
\end{aligned}
$$

The proof is given in Section 3

Corollary 2.2. Let $X$ be a complex space, $S$ an analytic subset in $X$, and $\varphi$ : $X \rightarrow[-\infty, \infty)$ an upper semicontinuous function on $X$. Then the Hartogs domain $\Omega_{\varphi}(X)$ is hyperbolic modulo $\widetilde{S}$ if and only if $X$ is hyperbolic modulo $S$ and $\varphi$ is locally bounded (below) on $X \backslash S$.

The situation for tautness is a bit more complicated, at least in the case of complex spaces.

Theorem 2.3. Let $X$ be a complex space and $S$ be an analytic subset in $X$. Then:

(i) If $\Omega_{H}(X)$ is taut modulo $\widetilde{S}$, then $X$ is taut modulo $S$ and $\log H$ is continuous plurisubharmonic on $X \backslash S \times \mathbb{C}^{m}$.

(ii) Furthermore if $X$ is a connected and locally irreducible complex space and $S$ a (proper) analytic subset, then in addition $\log H$ is plurisubharmonic on $X \times \mathbb{C}^{m}$.

(iii) Conversely, if $X$ is taut modulo $S, H$ is continuous on $X \backslash S \times \mathbb{C}^{m}$ and $\log H$ is plurisubharmonic on $X \times \mathbb{C}^{m}$, then $\Omega_{H}(X)$ is taut modulo $\widetilde{S}$.

The proof is given in Section 4 
As above, an immediate corollary is obtained for Hartogs domains by observing that $H(z, w):=|w| e^{\varphi(z)}$ is continuous if and only if $\varphi$ is, and $\log H$ is plurisubharmonic if and only if $\varphi$ is.

When $X$ is a connected manifold, the theorem implies that $\Omega_{H}(X)$ is taut modulo $\widetilde{S}$ if and only if $X$ is taut modulo $S, H$ is continuous on $X \backslash S \times \mathbb{C}^{m}$ and $\log H$ is plurisubharmonic on $X \times \mathbb{C}^{m}$.

Implication (ii) does not hold for the more general case of a connected but reducible complex space, as the following shows.

Example 2.4. Let $X:=\left\{\left(z_{1}, z_{2}\right) \in \mathbb{C}^{2}: z_{1} z_{2}=0\right\}, S:=\left\{\left(z_{1}, z_{2}\right) \in \mathbb{C}^{2}: z_{2}=0\right\}$, and $\varphi\left(z_{1}, z_{2}\right):=\log \left|z_{2}\right|$. Then $\Omega_{\varphi}(X)$ is taut modulo $S$, but $\varphi \notin P S H(X)$.

Proof. Since $\varphi$ is identically $-\infty$ on an open set, it can't be plurisubharmonic. If a sequence $\left(F_{n}\right) \subset \operatorname{Hol}\left(\mathbb{D}, \Omega_{\varphi}(X)\right)$ is not compactly divergent modulo $\widetilde{S}$, it is easy to see that $F_{n}(\mathbb{D}) \subset\left\{(z, w) \in \Omega_{\varphi}(X): z_{1}=0\right\}$. But then if $F_{n}=\left(0, g_{n}, h_{n}\right)$, its convergence is equivalent to that of $\left(g_{n}, h_{n}\right) \subset \operatorname{Hol}\left(\mathbb{D}, \Omega_{\varphi_{0}}(\mathbb{C})\right)$, where $\varphi_{0}\left(z_{2}\right)=\log \left|z_{2}\right|$. We know that $\left(g_{n}, h_{n}\right)$ is not compactly divergent modulo $\{0\}$, and Theorem 2.3 shows that $\Omega_{\varphi_{0}}(\mathbb{C})$ is taut modulo $\{0\}$, so a subsequence must converge on compacta.

\section{Hyperbolicity}

Recall that the Lempert function is defined as $\ell_{\Omega}(a, b)=\inf \left\{d_{\mathbb{D}}(0, \lambda): \exists \varphi \in\right.$ $\operatorname{Hol}(\mathbb{D}, \Omega), \varphi(0)=a, \varphi(\lambda)=b\}$ and that $\ell_{\Omega}(a, b) \leq k_{\Omega}(a, b)$.

Using the same argument as in the proof of Remark 3.1.7 and Proposition 3.1.10 in [9], it is easy to see the following.

Lemma 3.1. Let $\Omega=\Omega_{H}(X)$. Then $\ell_{\Omega}((z, 0),(z, w)) \leq d_{\mathbb{D}}(0, H(z, w))$ for any $(z, w) \in \Omega$, where $p$ is the Poincaré distance. Equality holds if $H \in P S H\left(X \times \mathbb{C}^{m}\right)$.

Proof of Theorem 2.1. ( $\Longrightarrow)$ Suppose $\Omega_{H}(X)$ is hyperbolic. Since $X$ is isomorphic to a closed complex subspace of $\Omega_{H}(X)$, we deduce that $X$ is hyperbolic. Next, we will show that $H$ verifies property (11). Otherwise, there would exist $\left\{z_{k}\right\}_{k \geq 1} \subset$ $X \backslash S$ with $\lim _{k \rightarrow \infty} z_{k}=z_{0} \in X \backslash S$ and $\left\{w_{k}\right\}_{k \geq 1} \subset \mathbb{C}^{m}$ with $\lim _{k \rightarrow \infty} w_{k}=w_{0} \neq 0$ such that $\lim _{k \rightarrow \infty} H\left(z_{k}, w_{k}\right)=0$. Without loss of generality, we may assume that $\left(z_{k}, w_{k}\right) \in \Omega_{H}(X)$. Then by Lemma 3.1, we have

$$
0 \leq k_{\Omega}\left(\left(z_{k}, 0\right),\left(z_{k}, w_{k}\right)\right) \leq d_{\mathbb{D}}\left(0, H\left(z_{k}, w_{k}\right)\right), \forall k \geq 1 .
$$

By letting $k$ go to $\infty$, we find that $k_{\Omega}\left(\left(z_{0}, 0\right),\left(z_{0}, w_{0}\right)\right)=0$. This contradicts the hyperbolicity modulo $\tilde{S}$ of $\Omega_{H}(X)$.

$(\Longleftarrow)$ To prove the converse, we consider the projection $\pi: \Omega_{H}(X) \rightarrow X$ given by $\pi(z, w)=z$. Let $U$ be a compact neighbourhood of $z_{0}$ in $X \backslash S$. Then $\bigcup_{z \in U} \Omega_{H}(z)$ is a bounded set in $\mathbb{C}^{m}$, where $\Omega_{H}(z):=\left\{w \in \mathbb{C}^{m}: H(z, w)<1\right\}$.

In fact, suppose that this property does not hold. Then $\exists\left\{z_{k}\right\}_{k \geq 1} \subset U,\left\{w_{k}\right\}_{k \geq 1}$ $\subset \mathbb{C}^{m}$ such that $\lim _{k \rightarrow \infty}\left\|w_{k}\right\|=\infty$ and $H\left(z_{k}, w_{k}\right)<1$. Put $w_{k}:=r_{k} u_{k}$ with $\left\|u_{k}\right\|=$ $1, \forall k \geq 1$; then $\left|r_{k}\right| \rightarrow \infty$ as $k \rightarrow \infty$. Passing to a subsequence, we may assume that $z_{k} \rightarrow z_{0}$ and $u_{k} \rightarrow u_{0} \neq 0$ as $k \rightarrow \infty$. Since $H\left(z_{k}, w_{k}\right)=\left|r_{k}\right| H\left(z_{k}, u_{k}\right)<1$, we 
have $\limsup H\left(z_{k}, u_{k}\right)=0$. This contradicts property (10). So, there exists $R>0$ $k \rightarrow \infty$

such that $\pi^{-1}(U) \subset U \times \bigcup_{z \in U} \Omega_{H}(z) \subset U \times B(0, R)$. Therefore, $\pi^{-1}(U)$ is hyperbolic too. By Eastwood's theorem [5] we conclude the proof.

\section{TAutness}

\section{Proof of Theorem 2.3.}

Proof of (i). Since $X$ is isomorphic to a closed complex subspace of $\Omega_{H}(X)$, we deduce that $X$ is taut modulo $S$. We now show that $H$ is continuous on $X \backslash S \times \mathbb{C}^{m}$. Otherwise, there would exist $r>0,\left\{\left(z_{k}, w_{k}\right)\right\}_{k \geq 1} \subset X \backslash S \times \mathbb{C}^{m}$ such that

$$
\left\{\left(z_{k}, w_{k}\right)\right\} \rightarrow\left(z_{0}, w_{0}\right), z_{0} \in X \backslash S \text { and } H\left(z_{k}, w_{k}\right)<r<H\left(z_{0}, w_{0}\right), \forall k \geq 1 .
$$

For each $k \geq 1$, we define the holomorphic mapping $f_{k}: \mathbb{D} \rightarrow \Omega_{H}(X)$ given by $f_{k}(\lambda)=\left(z_{k}, \frac{\lambda w_{k}}{r}\right)$. It is clear that $f_{k}(0)=\left(z_{k}, 0\right) \rightarrow\left(z_{0}, 0\right) \in \Omega_{H}(X) \backslash \tilde{S}$. Since $\Omega_{H}(X)$ is taut modulo $\tilde{S}$, by passing to a subsequence if necessary, we may assume that $f_{k}$ converges locally uniformly on $\mathbb{D}$ to a holomorphic mapping $f \in$ $\operatorname{Hol}\left(\mathbb{D}, \Omega_{H}(X)\right)$. It is easy to see that $f(\lambda)=\left(z_{0}, \frac{\lambda w_{0}}{r}\right)$. Hence

$$
\frac{|\lambda|}{r} H\left(z_{0}, w_{0}\right)=H\left(z_{0}, \frac{\lambda w_{0}}{r}\right)<1, \forall \lambda \in \mathbb{D} .
$$

This implies that $H\left(z_{0}, w_{0}\right)<\frac{r}{|\lambda|}, \forall \lambda \in \mathbb{D}$, and hence $H\left(z_{0}, w_{0}\right) \leq r$. This is a contradiction.

It remains to show that $\log H$ is plurisubharmonic.

According to the theorem of Fornaess and Narasimhan [6], it suffices to show that $u(z):=\log H \circ g(z)=\log H\left(g_{1}(z), g_{2}(z)\right)$ is subharmonic for every $g=\left(g_{1}, g_{2}\right) \in$ $\operatorname{Hol}\left(\mathbb{D}, X \backslash S \times \mathbb{C}^{m}\right) \cap C\left(\overline{\mathbb{D}}, X \backslash S \times \mathbb{C}^{m}\right)$. Suppose the contrary. Then $\exists z_{0} \in \mathbb{D}, r>0$ such that $\bar{D}\left(z_{0}, r\right) \subset \mathbb{D}$ and a harmonic function $h$ such that $h(z) \geq u(z)$ for any $z=z_{0}+r e^{i \theta}, \forall \theta \in \mathbb{R}$, but $u\left(z_{0}\right)>h\left(z_{0}\right)$. Let $\tilde{h}$ denote a harmonic conjugate to $h$. We have

$$
u(z)-h(z)=\log H\left(g_{1}(z), e^{-h(z)-i \tilde{h}(z)} g_{2}(z)\right) \leq 0, \forall z=z_{0}+r e^{i \theta},
$$

and $u\left(z_{0}\right)-h\left(z_{0}\right)=\varepsilon_{0}>0$.

For any $n \geq 1$, we set

$$
\varphi_{n}(\lambda):=\left(g_{1}(z), e^{-h(z)-i \tilde{h}(z)-\varepsilon_{0}-\frac{1}{n}} g_{2}(z)\right),
$$

where $z \in \bar{D}\left(z_{0}, r\right)$. Then $\varphi_{n} \in \operatorname{Hol}\left(D\left(z_{0}, r\right), \Omega_{H}(X)\right) \cap C\left(\bar{D}\left(z_{0}, r\right), \Omega_{H}(X)\right)$, $\bigcup_{n=1}^{\infty} \varphi_{n}\left(\partial D\left(z_{0}, r\right)\right) \Subset \Omega_{H}(X)$, and $\varphi_{n}(0)$ tend to a boundary point. This contradicts the tautness of $\Omega_{H}(X)$.

Proof of (ii). By the removable singularity theorem for plurisubharmonic functions [10, Theorem 2.9.22], since $\log H$ is locally bounded above in $X \times \mathbb{C}^{m}$ and plurisubharmonic in $X \backslash S \times \mathbb{C}^{m}$, it can be extended across $\tilde{S}$ to a function $\log \hat{H} \in$ $P S H\left(X \times \mathbb{C}^{m}\right)$ given, for $\left(z_{0}, w_{0}\right) \in \tilde{S}$, by

$$
\log \hat{H}\left(z_{0}, w_{0}\right):=\limsup _{(z, w) \rightarrow\left(z_{0}, w_{0}\right),(z, w) \notin \tilde{S}} \log H(z, w) .
$$


We claim that $\hat{H}=H$. Since $H$ is upper semicontinuous on $X \times \mathbb{C}^{m}$, this conclusion can only fail if there exists $\left(z_{0}, w_{0}\right) \in \tilde{S}$ such that

$$
H\left(z_{0}, w_{0}\right)>\limsup _{(z, w) \rightarrow\left(z_{0}, w_{0}\right),(z, w) \notin \tilde{S}} H(z, w) .
$$

Lemma 4.1. Under the hypotheses of Theorem 2.3 (ii), there exists an analytic disk $f$ in $X \times \mathbb{C}^{m}$ such that $f(0)=\left(z_{0}, w_{0}\right), f(\mathbb{D}) \not \subset \tilde{S}$.

Proof. It is enough to find a disk $f_{0}$ in $X$ with $f_{0}(0)=x_{0}$ and set $f:=\left(f_{0}, w_{0}\right)$. When $X$ is a manifold, we can go to a coordinate patch and use an affine analytic disk, for instance.

In general, since $X$ is locally irreducible, $S$ must be of codimension at least one; see for instance [7, Theorem C14, Ch. III, p. 115]. Let $(M, \pi)$ be a desingularization of $X$ [8, 2]; i.e., $M$ is a complex manifold, $\pi$ is a holomorphic map, $\pi^{-1}\left(X_{\text {sing }}\right)$ is a hypersurface in $M$, and $\pi$ is a biholomorphism from $M \backslash \pi^{-1}\left(X_{\text {sing }}\right)$ to $X \backslash X_{\text {sing }}$.

Let $p_{0}$ be such that $\pi\left(p_{0}\right)=x_{0}$. Since $\pi^{-1}(S)$ must be an analytic set of codimension at least one, there is an analytic disk $f_{1}$ in $M$ such that $f_{1}(0)=p_{0}$, $f(\mathbb{D}) \not \subset \pi^{-1}(S)$. The $\operatorname{disc} f_{0}:=\pi \circ f_{1}$ will do.

Then $f^{-1}(\tilde{S})$ must be a discrete subset of $\mathbb{D}$, and reducing the disk we may assume that $f^{-1}(\tilde{S})=\{0\}$ and $\sup _{0<|\zeta|} H(f(\zeta))=H\left(z_{0}, w_{0}\right)-\delta, \delta>0$. The proof then proceeds essentially as that of (i). We have a contradiction.

Proof of (iii). Assume that $X$ is taut modulo $S$ and $\log H$ is continuous on $X \backslash S \times$ $\mathbb{C}^{m}$ and plurisubharmonic on $X \times \mathbb{C}^{m}$. We now show that $\Omega_{H}(X)$ is taut modulo $\widetilde{S}$.

Consider the projection $\pi: \Omega_{H}(X) \rightarrow X$ defined by $\pi(x, z)=x$. We now prove, for each $x \in X \backslash S$, that there exists an open neighbourhood $U$ of $x$ in $X \backslash S$ such that $\pi^{-1}(U)$ is taut. Indeed, choose a hyperconvex neighbourhood $U$ of $x$ in $X \backslash S$. It is easy to see that $\pi^{-1}(U)=\left\{(u, z) \in U \times \mathbb{C}^{m}: H(z, w)<1\right\}=\Omega_{H}(U)$. Suppose that $\rho$ is a negative plurisubharmonic exhaustion function of $U$. Then $(u, z) \mapsto$ $\max (\rho(u), \log H(u, z))$ is also a negative plurisubharmonic exhaustion function of $\Omega_{H}(U)$. Thus, $\Omega_{H}(U)$ is hyperconvex. By a theorem of Sibony [13] and [15], $\Omega_{H}(U)$ is taut. Thus, $\pi^{-1}(U)$ is taut.

Assume that a sequence $\left\{\widetilde{f}_{n}\right\} \subset \operatorname{Hol}\left(\mathbb{D}, \Omega_{H}(X)\right)$ is not compactly divergent modulo $\widetilde{S}$ in $\operatorname{Hol}\left(\mathbb{D}, \Omega_{H}(X)\right)$. From now on, denote $\widetilde{X}:=\Omega_{H}(X) \backslash \widetilde{S}$.

Without loss of generality, we may assume that there exist a compact set $K \subset \mathbb{D}$ and a compact set $L \subset \widetilde{X}$ such that $\widetilde{f}_{n}(K) \cap L \neq \emptyset$ for $n \geq 1$. For each $n \geq 1$, there exists $z_{n} \in K \subset \mathbb{D}$ such that $\tilde{f}_{n}\left(z_{n}\right) \in L$. Since $K$ and $L$ are compact sets, by taking subsequences if necessary, we may assume that $\left\{z_{n}\right\} \subset K \subset \mathbb{D}$ such that $z_{n} \rightarrow z_{\infty} \in$ $K \subset \mathbb{D}$ and $\widetilde{f}_{n}\left(z_{n}\right) \rightarrow \widetilde{p} \in L \subset \widetilde{X}$. It is easy to see that $\left\{f_{n}:=\pi \circ \widetilde{f}_{n}\right\} \subset \operatorname{Hol}(\mathbb{D}, X)$ is not compactly divergent modulo $S$ in $H o l(\mathbb{D}, X)$. Since $X$ is taut modulo $S$, we may assume that $\left\{f_{n}\right\}$ converges uniformly to a mapping $F \in \operatorname{Hol}(\mathbb{D}, X)$. Obviously, $\pi\left(\tilde{f}_{n}\left(z_{n}\right)\right) \rightarrow \pi(\widetilde{p})$ and $\pi\left(\tilde{f}_{n}\left(z_{n}\right)\right)=\pi \circ \tilde{f}_{n}\left(z_{n}\right)=f_{n}\left(z_{n}\right) \rightarrow F\left(z_{\infty}\right)$ as $n \rightarrow \infty$. Therefore, we can let $p=\pi(\widetilde{p})=F\left(z_{\infty}\right)$. 
Since $\widetilde{p} \in \widetilde{X}, p=\pi(\widetilde{p}) \notin S$. Then there exists an open neighbourhood $U$ of $p$ in $X \backslash S$ such that $\pi^{-1}(U)$ is taut. Taking an open neighbourhood $V \Subset F^{-1}(U)$ of $z_{\infty}$ in $\mathbb{D} \backslash F^{-1}(S)$ and since the sequence $\left\{f_{n}\right\}$ converges uniformly to a mapping $F$, we may assume that $f_{n}(V) \subset U$. This implies that $\tilde{f}_{n}(V) \subset \pi^{-1}(U)$ for every $n>1$.

Consider the compact subsets $K=\left\{z_{n}, n \in \mathbb{Z}_{+}\right\} \cup\left\{z_{\infty}\right\} \subset \mathbb{D}$ and $L=\left\{\widetilde{f}_{n}\left(z_{n}\right)\right\} \cup$ $\{\widetilde{p}\} \subset \tilde{X}$. Then $\tilde{f}_{n}(K) \cap L \neq \emptyset$ for all $n$, and hence the sequence $\left\{\left.\tilde{f}_{n}\right|_{V}\right\}$ is not compactly divergent modulo $\widetilde{S}$ in $\operatorname{Hol}\left(\mathbb{D}, \Omega_{H}(X)\right)$. The fact that $\pi^{-1}(U)$ is taut and $\widetilde{f}_{n}(V) \subset \pi^{-1}(U)$ imply that $\left\{\left.\widetilde{f}_{n}\right|_{V}\right\}$ converges uniformly to a mapping $\widetilde{F}$ in $\operatorname{Hol}\left(V, \pi^{-1}(U)\right)$.

Consider the family $\Gamma$ of all pairs $(W, \Phi)$, where $W$ is an open set in $\mathbb{D} \backslash F^{-1}(S)$ and $\Phi \in \operatorname{Hol}(W, \tilde{X})$ such that there exists a subsequence $\left\{\left.\widetilde{f}_{n_{k}}\right|_{W}\right\}$ of $\left\{\left.\widetilde{f}_{n}\right|_{W}\right\}$ which converges uniformly to mapping $\Phi$ in $\operatorname{Hol}(W, \tilde{X})$.

According to the proof above, we have $\Gamma \neq \emptyset$. We now consider the following order relation in the family $\Gamma:\left(W_{1}, \Phi_{1}\right) \leq\left(W_{2}, \Phi_{2}\right)$ if

(i) $W_{1} \subset W_{2}$ and

(ii) for any subsequence $\left\{\left.\tilde{f}_{n_{k}}\right|_{W_{1}}\right\}$ of $\left\{\left.\tilde{f}_{n}\right|_{W_{1}}\right\}$ that converges uniformly to mapping $\Phi_{1}$ in $\operatorname{Hol}\left(W_{1}, \tilde{X}\right)$, there exists a subsequence $\left\{\widetilde{f}_{n_{k_{l}}}\right\}$ of $\left\{\widetilde{f}_{n_{k}}\right\}$ such that the sequence $\left\{\left.\widetilde{f}_{n_{k_{l}}}\right|_{W_{2}}\right\}$ converges uniformly to mapping $\Phi_{2}$ in $\operatorname{Hol}\left(W_{2}, \widetilde{X}\right)$.

Assume that $\left\{\left(W_{\alpha}, \Phi_{\alpha}\right)\right\}_{\alpha \in \Lambda}$ is a well-ordered subset of $\Gamma$. Put $W_{0}=\bigcup_{\alpha \in \Lambda} W_{\alpha}$ and define a mapping $\Phi_{0} \in \operatorname{Hol}\left(W_{0}, \Omega_{H}(X) \backslash \widetilde{S}\right)$ given by $\left.\Phi_{0}\right|_{W_{\alpha}}=\Phi_{\alpha}$ for each $\alpha \in \Lambda$.

Take a sequence $\left\{\left(W_{i}, \Phi_{i}\right)\right\}_{i=1}^{\infty} \subset\left\{\left(W_{\alpha}, \Phi_{\alpha}\right)\right\}_{\alpha \in \Lambda}$ such that

$$
\left(W_{1}, \Phi_{1}\right) \leq\left(W_{2}, \Phi_{2}\right) \leq \cdots \text { and } W_{0}=\bigcup_{i=1}^{\infty} W_{i}
$$

By the definition of $\Gamma$, there exists a subsequence $\left\{\left.\widetilde{f}_{n}^{1}\right|_{W_{1}}\right\}$ of $\left\{\left.\widetilde{f}_{n}\right|_{W_{1}}\right\}$ such that $\left\{\left.\widetilde{f}_{n}^{1}\right|_{W_{1}}\right\}$ converges uniformly to the mapping $\Phi_{1}$ in $\operatorname{Hol}\left(W_{1}, \widetilde{X}\right)$. By the definition of the order relation on $\Gamma$, there exists a subsequence $\left\{\widetilde{f}_{n}^{2}\right\}$ of $\left\{\widetilde{f}_{n}^{1}\right\}$ which converges uniformly to $\Phi_{2}$ in $\operatorname{Hol}\left(W_{2}, \widetilde{X}\right)$.

By continuing this process, we get the sequence $\left\{\widetilde{f}_{n}^{k}\right\}$ such that $\left\{\widetilde{f}_{n}^{k}\right\} \subset\left\{\widetilde{f}_{n}^{k-1}\right\}$ for all $k \geq 2$ and $\left\{\left.\widetilde{f}_{n}^{k}\right|_{W_{k}}\right\}$ converges uniformly to $\Phi_{k}$ in $\operatorname{Hol}\left(W_{k}, \widetilde{X}\right)$. Thus, a diagonal sequence $\left\{\widetilde{f}_{k}^{k}\right\}$ converges uniformly to $\Phi_{0}$ in $\operatorname{Hol}\left(W_{0}, \widetilde{X}\right)$. Hence $\left(W_{0}, \Phi_{0}\right) \in \Gamma$ and the subset $\left\{\left(W_{\alpha}, \Phi_{\alpha}\right)\right\}_{\alpha \in \Lambda}$ of $\Gamma$ has a supremum.

By the Zorn lemma, there exists a maximal element $(W, \Phi)$ of the family $\Gamma$. Assume that $\left\{\left.\widetilde{f}_{n_{k}}\right|_{W}\right\}$ is a subsequence of $\left\{\left.\widetilde{f}_{n}\right|_{W}\right\}$ such that $\left\{\left.\widetilde{f}_{n_{k}}\right|_{W}\right\}$ converges uniformly to $\Phi$ in $\operatorname{Hol}(W, \widetilde{X})$.

We now show that $W=\mathbb{D} \backslash F^{-1}(S)$. Suppose that there exists $z_{0} \in \bar{W} \cap(\mathbb{D} \backslash$ $\left.F^{-1}(S)\right)$. Take an open neighbourhood $U$ of $F\left(z_{0}\right)$ in $X \backslash S$ such that $\pi^{-1}(U)$ is taut. Since $\left\{f_{n}\right\}$ converges uniformly to a mapping $F$ in $\operatorname{Hol}(\mathbb{D}, X)$, there exists an open neighbourhood $W_{0}$ of $z_{0}$ in $\mathbb{D} \backslash F^{-1}(S)$ such that $\pi \circ \widetilde{f}_{n}\left(W_{0}\right) \subset U$. Hence $\widetilde{f}_{n}\left(W_{0}\right) \subset \pi^{-1}(U)$ for all $n \geq 1$. 
Fix $z_{1} \in W_{0} \cap W$. Then the sequence $\left\{\tilde{f}_{n_{k}}\left(z_{1}\right)\right\}$ is convergent. Since $\operatorname{Hol}\left(W_{0}, \pi^{-1}(U)\right)$ is a normal family, $\left\{\left.\tilde{f}_{n_{k}}\right|_{W_{0}}\right\}$ converges uniformly to $\Phi_{0}$ in $\operatorname{Hol}\left(W_{0}, \pi^{-1}(U)\right)$. Thus $\left(W_{0}, \Phi_{0}\right) \in \Gamma$. This implies that $W_{0} \subset W$. Hence $W=\mathbb{D} \backslash F^{-1}(S)$.

Since $F^{-1}(S)$ is an analytic subset in the open unit disc $\mathbb{D}, F^{-1}(S)$ is a discrete set, and hence $F^{-1}(S)$ does not have any accumulation point in $\mathbb{D}$. Therefore, for each $z \in F^{-1}(S)$, there exists a number $0<r<1$ such that $B(z, r) \cap F^{-1}(S)=\{z\}$. Without loss of generality, we may assume that $\mathbb{D} \backslash F^{-1}(S)=\mathbb{D} \backslash\{0\}:=\mathbb{D}^{*}$ and the sequence $\left\{\left.\widetilde{f}_{n}\right|_{\mathbb{D}^{*}}\right\}$ converges uniformly to the map $\Phi \in \operatorname{Hol}\left(\mathbb{D}^{*}, \widetilde{X}\right)$.

We now rewrite functions $\tilde{f}_{n}$ and $\Phi$ as follows: $\tilde{f}_{n}(z)=\left(f_{n}(z), g_{n}(z)\right)$ for each $z \in \mathbb{D}$ and $\Phi(z)=(F(z), G(z))$ for each $z \in \mathbb{D}^{*}$. Since the sequence $\left\{g_{n}\right\}$ converges uniformly on compact subsets of $\mathbb{D}^{*}$ to $G \in \operatorname{Hol}\left(\mathbb{D}^{*}, \mathbb{C}^{m}\right)$, the maximum principle implies that it is a uniformly Cauchy sequence on compact subsets of $\mathbb{D}$ and therefore converges uniformly on compact subsets of $\mathbb{D}$ to $\tilde{G} \in \operatorname{Hol}\left(\mathbb{D}, \mathbb{C}^{m}\right)$. Denote $\tilde{\Phi}=$ $(F, \tilde{G}): \mathbb{D} \rightarrow X \times \mathbb{C}^{m}$. We now prove that $\tilde{\Phi} \in \operatorname{Hol}\left(\mathbb{D}, \Omega_{H}(X)\right)$. Since $\tilde{\Phi}\left(\mathbb{D}^{*}\right)=$ $\Phi\left(\mathbb{D}^{*}\right) \subset \Omega_{H}(X)$, it suffices to show that $\tilde{\Phi}(0) \in \Omega_{H}(X)$.

Indeed, since $H$ is plurisubharmonic on $X \times \mathbb{C}^{m}, H \circ \tilde{\Phi}$ is subharmonic on $\mathbb{D}$, and since $\Phi\left(\mathbb{D}^{*}\right) \subset \Omega_{H}(X)$, it is negative on $\mathbb{D}^{*}$. So it is negative on $\mathbb{D}$, i.e., $\tilde{\Phi}(\mathbb{D}) \subset \Omega_{H}(X)$. Hence $\Omega_{H}(X)$ is taut modulo $\widetilde{S}$.

\section{ACKNOWLEDGEMENT}

We would like to thank the anonymous referee for a lightning fast and thorough job, as well as for suggesting that a result analogous to Lemma 4.1 could be proved by resorting to the methods of [3], which proved quite inspiring.

\section{References}

1. Y. Adachi, On a Kobayashi hyperbolic manifold $N$ modulo a closed subset and its applications, Kodai Math. J. 30 (2007), 131-139. MR2319082 (2008h:32034)

2. E. Bierstone, P. D. Milman, Canonical desingularization in characteristic zero by blowing up the maximum strata of a local invariant, Invent. Math. 128 (1997), 207-302. MR.1440306 (98e:14010)

3. B. Drinovec Drnovšek, F. Forstnerič, Disc functionals and Siciak-Zaharyuta extremal functions on singular varieties, arXiv:1109.3947, to appear in Ann. Polon. Math.

4. Nguyen Quang Dieu and Do Duc Thai, Complete hyperbolicity of Hartogs domain, Manuscripta Math. 112 (2003), 171-181. MR2064914 (2005c:32004)

5. A. Eastwood, À propos des variétés hyperboliques complètes, C. R. Acad. Sci. Paris 280 (1975), 1071-1075. MR0414941 (54:3033)

6. J. Fornaess and R. Narasimhan, The Levi problem on complex spaces with singularities, Math. Ann. 248 (1980), 47-72. MR569410 (81f:32020)

7. R. C. Gunning, H. Rossi, Analytic Functions of Several Complex Variables, Prentice Hall, Englewood Cliffs, N.J. (1965). MR0180696 (31:4927)

8. H. Hironaka, Desingularization of complex-analytic varieties (in French), Actes du Congrès International des Mathématiciens (Nice, 1970), Tome 2, 627-631, Gauthier-Villars, Paris, 1971. MR0425170(54:13127)

9. M. Jarnicki and P. Pflug, Invariant Distances and Metrics in Complex Analysis, Walter de Gruyter, Berlin-New York (1993). MR.1242120 (94k:32039)

10. M. Klimek, Pluripotential Theory, Oxford University Press, New York, 1991. MR.1150978 (93h:32021)

11. S. Kobayashi, Hyperbolic Complex Spaces, Springer-Verlag, Berlin, 1998. MR1635983 (99m:32026) 
12. R. Remmert, Classical Topics in Complex Function Theory, Springer-Verlag, Berlin, 1998. MR.1483074 (98g:30002)

13. N. Sibony, A class of hyperbolic manifolds, Recent Developments in Several Complex Variables, Princeton Univ. Press 100 (1981), 357-372. MR627768 (83a:32022)

14. Do Duc Thai and P. J. Thomas, $D^{*}$-extension property without hyperbolicity, Indiana Univ. Math. J. 47 (1998), 1125-1130. MR.1665757 (99j:32012)

15. Do Duc Thai and Pham Viet Duc, On the complete hyperbolicity and the tautness of the Hartogs domains, Intern. J. Math. 11 (2000), 103-111. MR.1757893(2001g:32059)

16. Nguyen Van Trao and Tran Hue Minh, Remarks on the Kobayashi hyperbolicity of complex spaces, Acta Math. Vietnam. 34 (2009), 375-387. MR2583947 (2011a:32041)

Department of Mathematics, Hanoi National University of Education, 136 Xuan ThuY STR., HANOI, VIETNAM

E-mail address: ducthai.do@gmail.com

Université De Toulouse, UPS, INSA, UT1, UTM, Institut de Mathématiques De Toulouse, F-31062 Toulouse, France

E-mail address: pascal.thomas@math.univ-toulouse.fr

Department of Mathematics, Hanoi National University of Education, 136 Xuan ThuY STR., Hanoi, Vietnam

E-mail address: ngvtrao@yahoo.com

Department of Mathematics, Hanoi National University of Education, 136 Xuan ThuY STR., Hanoi, Vietnam

E-mail address: ducphuongma@gmail.com 